\title{
Diderot et la dynamique productive de l'esprit
}

\author{
MITIA RIOUX-BEAULNE
}

adresse de rattachement

Disons-le d'emblée : l'étude qui suit ne porte pas sur un problème typiquement diderotien, si l'on entend par là quelque chose que Diderot aurait délibérément thématisé dans les termes qui nous servent à le poser. Elle résulte en fait d'un effort pour réfléchir sur un aspect de la théorie de la connaissance en lien avec celle de l'art sur l'horizon dessiné par la pensée du philosophe. Cette problématique forme une espèce de foyer vers lequel convergent plusieurs motifs diderotiens, sorte de point d'entrée dans la philosophie de Diderot. L'existence de tels foyers constitue en un sens une raison de croire que malgré l'absence de système qui la caractérise, la pensée de Diderot peut à bon droit être regardée du point de vue de la cohérence de sa mise en œuvre. Celle-ci s'accommode de variations de vocabulaire et d'hésitations théoriques, source de tensions qu'il serait vain de vouloir dissoudre - ne serait-ce que parce qu'elles sont au principe de sa fécondité.

Il s'agit donc ici d'interroger la jonction entre ce que les penseurs de la période moderne n'hésitent pas à appeler des productions de l'esprit, c'està-dire les mécanismes cognitifs orientés vers l'élaboration d'un savoir et l'activité proprement créatrice de l'esprit orientée vers la production artistique. Jusqu'à un certain point, ce problème a rapport avec l'évolution sémantique des catégories relatives à l'invention ${ }^{1}$. Dominées au début de l'âge classique par leurs emplois rhétoriques et poétiques, ces catégories renvoient d'abord à une activité de discernement de l'esprit qui, dans le cadre d'une esthétique fondée sur l'imitation des Anciens, implique la capacité de choisir dans le " déjà-là » de la tradition ce qui est propre à produire l'effet qu'on recher-

1. Sur cette évolution, voir Jacinthe MARTEL, «De l'invention. Éléments pour l'histoire lexicologique et sémantique d'un concept : XVI ${ }^{\mathrm{e}} \mathrm{XX}^{\mathrm{e}}$ siècles ", Études françaises, XXVI, 1990/3, p. 29-49. Voir aussi Annie BECQ, "Splendeur et misère de l'imitation au siècle des Lumières ", in Ulla KöLving et Irène Passeron (dir.), Sciences, musiques, Lumières : mélanges offerts à Anne-Marie Chouillet, Ferney, Centre international d'étude du XVIII siècle, 2001, p. 385-391. 
che (preuves pour convaincre, figures émouvantes, etc.). Mais elles acquièrent peu à peu un sens différent au cours des XVII ${ }^{e}$ et XVIII ${ }^{e}$ siècles et commencent à désigner la faculté simultanée de découvrir et de créer quelque chose de nouveau. C'est au cœur même de ce flottement, dans les efforts pour justifier l'emploi d'un même terme pour ces deux opérations, autant que dans ceux pour les séparer clairement, que se noue une série de questions relatives à l'apparition d'une esthétique philosophique comme champ autonome de réflexion, à l'émergence d'une interrogation originale sur le rapport entre les arts mécaniques, les arts libéraux et les sciences, à la valorisation croissante de l'imagination comme faculté où s'enracine le pouvoir créatif de l'esprit, au développement enfin d'une réflexion sur le rôle des signes dans la formation des idées.

Dans cette configuration ambivalente de la théorie de l'invention, le problème est de déterminer le statut et la fonction des actes cognitifs qui y président. Tant que le rationalisme se conforte dans l'idée que la raison est ellemême productrice de connaissance, qu'elle est d'essence divine, que nous avons des idées innées, l'invention en philosophie se distingue de l'invention rhétorique et poétique surtout parce qu'elle s'exerce dans un domaine où la tradition ne fait pas autorité : lorsque Descartes entreprend de rejeter tout ce que contiennent les livres qu'il a étudiés, c'est qu'il juge que, la raison étant impartie à tous, le « déjà-là » de la philosophie est la raison elle-même.

Mais lorsque l'empirisme s'attaque à la théorie des idées innées - sans pour autant revenir à la théorie aristotélicienne des formes substantielles la question de la teneur des actes de l'esprit est posée. Chez Locke existe encore une possibilité de hiérarchiser les propriétés découvertes par voie de réflexion grâce à la distinction entre qualités premières et qualités secondes, les productions de l'esprit relevant d'une conception représentationnelle des idées - encore qu'en dernière analyse celles-ci ne contentent que notre désir de conservation -, mais le scepticisme et l'épicurisme modernes, couplés au nominalisme, tendent à ne voir dans les concepts que l'expression d'actes cognitifs. La connaissance est alors une véritable construction. Comme l'indique Annie Becq, à propos de la réplique gassendiste à la gnoséologie cartésienne, connaître ne s'entend plus comme une contemplation de substances, mais véritablement comme une construction de systèmes de concepts :

C'est dans la perspective ouverte par ce nominalisme conséquent qu'on aperçoit la possibilité de penser le matérialisme, non élémentaire, de la production des signes, du travail et des institutions, fondé sur une conscience lucide de la contingence et de la facticité universelles [menant à] la dénonciation de 
la connaissance-vision et de l'inventaire empirique, ouvrant la possibilité de la conception de la connaissance comme production ${ }^{2}$.

Néanmoins, tant que ce nominalisme demeure suspendu à un dualisme résiduel, ce que nous tenterons de montrer, la construction est pensée comme reconstruction, reproduction du système de la nature dans des concepts. Il nous semble voir, dans la pensée de Diderot, une sorte de tentative de penser en dehors d'un tel schème.

Le terme même de production de l'esprit connaît un certain nombre d'emplois dans la période moderne qui en font un lieu théorique complexe: certaines lignes de force fondamentales en circonscrivent néanmoins l'étendue. Trois exemples serviront ici à expliciter ce qui est en jeu ${ }^{3}$.

\section{Productions de l'esprit, invention et découverte}

\section{Dans son Novum Organum, Bacon écrit :}

Les productions de l'esprit [generationes mentis] et de la main paraissent extrêmement nombreuses, à considérer livres et travaux. Mais toute cette variété tient à une rare subtilité et à l'exploitation d'un petit nombre de choses déjà connues, elle ne tient pas au nombre des axiomes.

Bien plus, toutes les œuvres inventées à ce jour doivent plus au hasard et à la simple expérience qu'aux sciences : car les sciences qui sont les nôtres aujourd'hui ne sont rien d'autre que certaines combinaisons de découvertes antérieures, et non des moyens d'invention et des indications pour de nouvelles œuvres ${ }^{4}$.

Dans ce contexte, les productions de l'esprit désignent les œuvres qui résultent de l'invention, définition qui, dans sa généralité, serait tout aussi acceptable pour un poète, un rhéteur que pour le philosophe-de même que, s'agissant des productions de la main, elle conviendrait aussi au «mécani-

2. Annie BEcQ, Genèse de l'esthétique française moderne, Paris, Albin Michel, 1994 [Ospedaletto, Pacini editore, 1984], p. 127-128.

3. Nous ne prétendons en aucun cas substituer un tel schématisme à ce que pourrait révéler une véritable enquête lexicographique : ce que nous avons en vue, c'est la reconstitution d'un contexte théorique à l'intérieur duquel les convergences et les divergences philosophiques deviennent apparentes, et ce, indépendamment de leurs filiations réelles. Ce n'est donc pas de l'histoire d'un concept dont nous nous efforçons de rendre compte, mais de l'histoire d'un problème - problème qui peut bien ne pas revenir en propre aux penseurs que nous abordons et n'être qu'un effet de notre lecture.

4. Francis BACON, Novum Organum (introduction, traduction et notes de M. Malherbe et J.-M. Pousseur), Paris, Presses universitaires de France, 1986, Livre I, § 7-8, p. 102. 
cien ». L'invention, comme catégorie méthodologique, est le processus fondamental qui assure la production du discours et des objets. Cependant, la banalité du propos ne doit pas faire écran à une difficulté d'emblée perceptible: même si ce qui est projeté par la refonte baconienne de la théorie de l'invention est de fournir «des indications pour de nouvelles œuvres", il doit être évident que ces nouvelles ouvres doivent constituer de nouvelles découvertes.

Ce qui se joue, dès lors, c'est l'assimilation par la science d'une catégorie oratoire: tant que l'invention demeure procédure de sélection des pensées qui doivent constituer un discours, le fait qu'elle soit essentiellement fondée sur des « combinaisons de découvertes antérieures " ne constitue pas un problème: les règles de la poétique qui dominent au moins jusqu'à la fin du XVII siècle, repliant l'invention sur l'imitation des Anciens, exigent précisément que la nouveauté des œuvres soit restreinte à ses composantes discursives ${ }^{5}$. La valorisation de l'invention comme partie la plus importante de la poétique n'implique en aucun cas la valorisation de la découverte - qui suppose, d'ailleurs, la remise en question de ce qui est avéré, attitude qui convient mal au règne de la scolastique. Lorsque Bacon, et Descartes à sa suite, tentent d'imprimer cette nouvelle orientation à la théorie de l'invention en en faisant une théorie de la méthode menant à de nouvelles découvertes, ils ébranlent par le fait même le sens tenu pour évident de ce que constitue une production de l'esprit: celle-ci n'est pas, à proprement parler, un discours, mais ce que ces discours représente, c'est-à-dire, justement, ce qui est conçu ou produit par l'esprit au terme du processus d'invention/découverte. Production donc qui fait apparaître la nature dans sa productivité, qui la réfléchit et en expose les mécanismes.

Dans la fameuse règle IV des Règles pour la direction de l'esprit, Descartes est ainsi amené, on s'en souviendra, à confondre la méthode d'invention ${ }^{6}$ avec sa Mathématique universelle, science purement déductive, qui, procédant de ses "semences de vérités " déposées par la nature dans les esprits humains, ne fait que déployer ce qui est analytiquement compris dans la raison divine qui donne son uniformité aux lois de la nature, et à laquelle

5. " La tâche du poète est donc bien de puiser sa matière poétique dans le déjà-là et non de l'inventer de toutes pièces. Au sein de cette esthétique de l'imitation, c'est moins en fonction de la nouveauté du sujet que par rapport à son renouvellement (au niveau de la disposition et de l'élocution) que peut s'opérer l'invention poétique. » (Jacinthe MarTel, " De l'invention... ", op. cit., p. 33.)

6. Il est remarquable de voir comment Descartes, dans ces pages, préserve vivante l'origine du terme d'invention par des références aux artisans, aux " machines ", etc. 
la raison humaine peut accéder ${ }^{7}$. En ce sens, l'activité productrice de l'esprit résultant de l'analyse n'est productrice que du point de vue du sujet qui amène graduellement à sa propre conscience ce qui est présent en lui, mais "que la lecture ou l'audition quotidiennes de tant d'erreurs diverses étouffent en [lui] ». Elle ne produit pas quelque chose de nouveau, mais fait voir cette chose. Le passage d'une catégorie artistique dans le champ de la théorie de la connaissance génère donc une sorte de paradoxe de l'invention.

Le caractère de production de ce qui est conçu n'est tel que pour l'esprit subjectif qui découvre, puisque ce qu'il produit, c'est ce qui était déjà là, sous ses yeux, mais recouvert et invisible. Dès lors, le discours qui représente cette production n'a pour fonction que de représenter pour autrui. Il ne s'agit pas de persuader - ce qui disqualifie toute procédure de recherche de preuves -, mais simplement de faire voir. Les productions de l'esprit ne désignent pas proprement les «œuvres nouvelles » que le Novum Organum semble dans un premier temps appeler de ses vœux, mais les seuls concepts et systèmes de concepts qui résultent de l'activité cognitive. Les œuvres auxquelles se destine ce nouvel art de penser sont des imitations de la nature dans le matériau de la pensée.

En régime cartésien, une telle possibilité pourrait signifier avant tout: non des imitations de la nature subsistante, matérielle, mais des idées telles qu'elles se trouvent dans l'esprit divin qui préside à la création ${ }^{8}$. En ce sens, on comprend que les productions de l'esprit, en tant que fruits d'une recherche de la vérité, pourront être interprétées par un cartésien notoire Malebranche - comme visions en Dieu ${ }^{9}$. Surtout, il importe à Descartes de distinguer entre la connaissance à laquelle nous parvenons à l'issue d'un tel travail de production/découverte et un autre type de production, celui-là même dont vient ce qui n'était pas déjà là: les imaginations. Dans celles-ci,

7. René Descartes, Règles pour la direction de l'esprit (traduction de J. Sirven), Paris, Vrin, 1972, p. 24.

8. Jusqu'à un certain point, ce qui est en cause ici, c'est la tentative cartésienne, dans la Seconde méditation de fonder sa théorie des concepts sur la distinction entre idée formelle et idée objective, laquelle permet de faire l'économie de l'ontologie scolastique des formes substantielles se traduisant psychologiquement en « espèces » tirées de la sensation, tout en préservant un fondement "réaliste » à son épistémologie. Sur ce point, la théorie cartésienne des idées fait écho à de nombreuses tentatives similaires chez ses contemporains. Voir, à ce sujet: Roger Ariew, Marjorie Grene, "Ideas, in and before Descartes ", Journal of the History of Ideas, vol. LVI, 1995, 1, p. 87-106.

9. Voir André RoBinet, Système et existence dans l'œuvre de Malebranche, Paris, Vrin (Bibliothèque d'histoire de la philosophie), 1965, p. 218-224 et 242-254; voir aussi Andrew Pessin, "Malebranche on Ideas », Canadian Journal of Philosophy, vol. 34, 2, juin 2004, p. 241-287. 
l'âme est soit active (c'est-à-dire qu'elle agit sous l'égide de la volonté et de l'entendement), soit passive (mue par l'action du corps: nerfs ou « cours fortuit des esprits animaux " ${ }^{10}$ ). Passive, elle est alors soumise à des illusions, songes, pensées erratiques, etc. ${ }^{11}$; mais active, elle conçoit alors un objet particulier selon des règles qui, parce qu'elles sont données par l'entendement, imitent les lois de la nature. Si la production humaine peut alors être appelée imitation de la nature, ce n'est pas en tant que représentation, mais en tant qu'imitation de l'activité productrice de la nature, ou plutôt en tant qu'imitation de l'activité créatrice de l'esprit de Dieu ${ }^{12}$. Suivant ce schéma, la production mécanicienne se trouve ainsi expliquée selon des termes qui font écho à la théorie traditionnelle de la production artistique. Le génie reposerait sur un enthousiasme défini en termes d'inspiration divine - l'acte de création poétique serait une sorte de réplique de l'acte de création par Dieu: organisation de la matière à partir d'une idée qui, elle, n'est pas une production, mais une réplique. La méthode, cependant, permettrait d'accéder aux règles de la production, assurant la maîtrise de ce qui n'a, jusqu'à présent, produit qu'un «fruit spontané » dû au hasard ${ }^{13}$.

La position de Diderot sur la question de l'imagination peut, ici, servir de pivot pour montrer comment sa pensée s'écarte de ce genre d'approche. Deux points retiennent l'attention.

(1) D'abord, la théorie diderotienne de la connaissance fait de la seule sensation le critère de la connaissance vraie: il n'y a de connaissance que là où il y a saisie actuelle par l'esprit. Une idée est donc d'autant plus distincte qu'elle est vive dans la sensation même, c'est-à-dire qu'elle repose sur la perfectibilité de notre puissance perceptive. Diderot substitue donc le raffinement de la sensibilité à l'analyse comme faculté productrice de connaissance. L'article IgNORAnce de l'Encyclopédie dira:

Si nous considérons en premier lieu que les notions que nous avons par nos facultés n'ont aucune proportion avec les choses mêmes, puisque nous n'avons

10. René Descartes, Les Passions de l'âme (introduction, notes, bibliographie et chronologie par P. D’Arcy), Paris, GF-Flammarion, 1996, § 26, p. 116.

11. Ibid., $\S 21$, p. 113.

12. En ce sens, l'imagination chez Descartes n'est pas une puissance propre-contrairement à ce qu'elle sera chez Pascal ou Malebranche. Elle est, beaucoup plus, un lieu où l'activité de l'esprit peut se manifester en produisant des figures. La Sixième Méditation sera claire sur ce point. Il y a définitivement chez Descartes une conception positive de l'imagination. Voir sur ce point Pierre Guenancia, «Le critique cartésienne des critiques de l'imagination », in Cynthia Fleury (dir.), Imagination, imaginaire, imaginal, Paris, PUF (Débats), 2006, p. 43-76.

13. René Descartes, Règles pour la direction de l'esprit, op. cit., " Règle IV », p. 18-21. 
pas une idée claire $\&$ distincte de la substance même qui est le fondement de tout le reste, nous reconnoîtrons aisément combien peu nous pouvons avoir de notions certaines; \& sans parler des corps qui échappent à notre connoissance, à cause de leur éloignement, il y en a une infinité qui nous sont inconnus à cause de leur petitesse. Or, comme ces parties subtiles qui nous sont insensibles, sont parties actives de la matiere, \& les premiers matériaux dont elle se sert, \& desquels dépendent les secondes qualités \& la plûpart des opérations naturelles, nous sommes obligés, par le défaut de leur notion, de rester dans une ignorance invincible de ce que nous voudrions connoître à leur sujet, nous étant impossible de former aucun jugement certain, n'ayant de ces premiers corpuscules aucune idée précise \& distincte.

S'il nous étoit possible de connoître par nos sens ces parties déliées \& subtiles, qui sont les parties actives de la matiere, nous distinguerions leurs opérations méchaniques avec autant de facilité qu'en a un horloger pour connoître la raison pour laquelle une montre va ou s'arrête. Nous ne serions point embarassés d'expliquer pourquoi l'argent se dissout dans l'eau-forte, \& non point dans l'eau régale; au contraire de l'or, qui se dissout dans l'eau régale, \& non pas dans l'eau-forte. Si nos sens pouvoient être assez aigus pour appercevoir les parties actives de la matiere, nous verrions travailler les parties de l'eauforte sur celles de l'argent, \& cette méchanique nous seroit aussi facile à découvrir, qu'il l'est à l'horloger de savoir comment, \& par quel ressort, se fait le mouvement d'une pendule ${ }^{14}$.

Cette emphase sur la perception sensible comme foyer de la découverte permet à Diderot de réinterpréter ce paradoxe de l'invention en faisant fond sur l'idée que la nouveauté de ce qui est découvert découle de son caractère de production, elle est un effet de la structuration du réel dans le sujet. En effet, l'une des conséquences du matérialisme de Diderot est la négation de la réalité des individus en dehors du sujet percevant:

Hemsterhuis:] Les premiers signes naturels sont les effets de l'objet sur l'organe. [Diderot:] Je n'entends pas. L'effet de l'objet sur l'organe est l'objet même, et non le signe de l'objet ou de la sensation ou de l'idée ${ }^{15}$.

Affirmant qu'il n'y a pas de distinction entre l'effet de l'objet et l'objet même, Diderot ruine le principe suivant lequel ce qui est visé par l'entendement dépasse le pouvoir de représentation. La sensation n'est pas une perception confuse de quelque « chose en soi » visé par la conscience, mais pure

14. Article Ignorance [Diderot], Encyclopédie, VIII, p. 649. Comparer avec la Réfutation d'Helvétius (Versini, I, p. 815) : «Une sensation fortuite n'est qu'un fait de plus; mais une sensation produite par un organe exquis et prodigieux est une multitude prodigieuse de faits; c'est la réunion du télescope et du microscope. "

15. Observations sur Hemsterhuis, dans Euvres (L. Versini éd.), Paris, Robert Laffont, 1994-1997, I, p. 696 - à l'avenir, les références à l'œuvre de Diderot renvoient toujours à cette édition, notée Versini, [volume, page]. 
chaîne de modifications se résolvant en une conscience d'objet. Ce qui fait de cette chaîne de modifications - "tableau mouvant " - une "sensation totale instantanée ", c'est que ces modifications convergent et se mettent en rapport dans une organisation, qu'elles s'unissent et se constituent en objet pour cette organisation ${ }^{16}$. Ce qui la fait objet, c'est qu'elle est un effet objectif ${ }^{17}$. L'opération qui fait passer le divers de la sensation à l'état d'objet ne résulte pas d'une activité réflexive, mais de la manière même dont les sensations s'organisent dans l'esprit, par une «spontanéité » de l'organisation qui, parce qu'elle est activité (sensibilité, propriété de la matière), produit des synthèse, assemble, divise, met en ordre factice ce divers ${ }^{18}$.

L'objet n'est donc ce qu'il est que par le biais de cette facticité immédiate que notre organisation imprime dans la mouvance permanente qui l'agite: l'individualité de l'objet est un concept faux ${ }^{19}$. Contre Condillac, qui donne une portée ontologique à l'unité conçue de l'objet sur la base de la réflexivité que confère le privilège du toucher ${ }^{20}$, Diderot dépasse même le fonde-

16. On aura reconnu les expressions de la Lettre sur les sourds et muets, Versini, IV, p. 2930: «autre chose la sensation totale instantanée de cet état, autre chose l'attention successive et détaillée que nous sommes forcés d'y donner pour l'analyser, la manifester et nous faire entendre. Notre âme est un tableau mouvant d'après lequel nous peignons sans cesse. "

17. On ne doutera pas que c'est l'assise physiologique de la pensée de Diderot qui est au fondement de cette idée. François Duchesneau ("Diderot et la physiologie de la sensibilité », Dix-huitième siècle, XXXI, 1999, p. 214) le remarque: "l'idée se dégage alors d'une théorie physiologique de l'homme, organisme supérieur issu d'une combinatoire de parties organiques. Ces parties convergent dans leurs interrelations dynamiques de façon à produire un effet global complexe, correspondant à l'unité indéfiniment variée du moi psychologique. Le psychisme correspond alors à un ensemble de phénomènes qui émergent d'organes divers interagissant selon des rapports immanents de sensibilité. Diderot en arrive ainsi à concevoir que la 'médecine physique' pourrait s'appliquer non seulement au corps, mais aussi aux âmes, puisque les états perceptifs et sensitifs sont tous des états corporels. "

18. «Voilà donc nos besoins, et l'exercice le plus immédiat de nos facultés qui conspirent aussitôt que nous naissons à nous donner des idées d'ordre, d'arrangement, de symétrie, de mécanisme, de proportion, d'unité; toutes ces idées viennent des sens et sont factices [...] Ces notions sont expérimentales comme toutes les autres. " Traité du beau, Versini, IV, p. 97-98. L'Encyclopédie (VI, p. 360) définit «factice» comme « qui est fait par art, qui n’est point naturel ».

19. «Et vous parlez d'individus, pauvres philosophes! [...] Que voulez-vous donc dire avec vos individus? Il n'y en a point, non, il n'y en a point... Il n'y a qu'un seul grand individu, c'est le tout. Dans ce tout, comme dans une machine, dans un animal quelconque, il y a une partie que vous appellerez telle ou telle: mais quand vous donnerez le nom d'individu à cette partie du tout, c'est par un concept aussi faux que si, dans un oiseau, vous donniez le nom d'individu à l'aile, à une plume de l'aile [...] Qu'est-ce qu'un être?... La somme d'un certain nombre de tendances [...] La vie, une suite d'actions et de réactions... Vivant, j'agis et je réagis en masse... mort, j'agis et je réagis en molécules. " (Le Rêve de d'Alembert, Versini, I, p. 636-637. [Nous soulignons])

20. Voir, à ce sujet: Bernard BAERTSCHI, Conscience et réalité. Études sur la philosophie française au XVIII ${ }^{e}$ siècle, Genève, Droz (Bibliothèque des Lumières), 2005, p. 81-89. 
ment ontologique traditionnel du nominalisme (l'idée qu'il n'existe que des individus).

À la multiplication des existants sans nécessité que représentait le dualisme de la métaphysique d'inspiration chrétienne, Diderot finit par opposer un tableau des existants et des principes constitutifs extrêmement épuré. Ce qui est: des atomes. Premier principe: les indiscernables (ce que Diderot appelle les deux grains de sable de Leibniz). Tous les atomes sont différents. La matière est fondamentalement hétérogène. Deuxième principe: la continuité, la liaison universelle. Tout se tient dans la nature, il n'y a pas de saut. D'où une remarquable économie métaphysique des individus: le tout est le seul individu. Il est indivisible, il a seul une existence propre et distincte. Toutes les autres choses ne sont jamais que des parties du tout, auxquelles on peut bien donner des noms, mais qui ne sont jamais distinctes (au sens de «séparables ") du tout, pas plus que l'aile n'est distincte de l'oiseau auquel elle appartient. Nous appartenons au tout ${ }^{21}$.

Ce qui importe, ici, c'est de noter qu'il suit de cette position que tout objet (individu), avant d'être faux, est un concept, c'est-à-dire l'effet d'une réification et d'une unification d'une chaîne de modifications dans une forme objectale, laquelle a lieu dans l'imagination.

(2) Cela permet de comprendre ceci: chez Diderot plus que chez tout autre, l'analyse ne constitue une augmentation de connaissance que si elle coordonne une décomposition en retour vers le sensible:

Demandez [au philosophe] par exemple: qu'est-ce que la justice? Et vous serez assez convaincu qu'il ne s'entendra lui-même que quand, la connaissance se portant de son âme vers les objets par le même chemin qu'elle y est venue, il imaginera deux hommes conduits par la faim vers un arbre chargé de fruits; l'un monté sur l'arbre, et cueillant; et l'autre s'emparant, par la violence, du fruit que le premier a cueilli. Alors il vous fera remarquer les mouvements qui se manifesteront en eux; les signes du ressentiment d'un côté, les symptômes de la crainte de l'autre; celui-là se tenant pour offensé, et l'autre se chargeant lui-même du titre d'odieux offenseur.

Si vous faites la question à un autre, sa dernière réponse se résoudra en un tableau. Autant de têtes, autant de tableaux différents peut-être: mais tous représenteront deux hommes éprouvant dans un même instant des impressions contraires; produisant des mouvements opposés; ou poussant des cris inarticulés et sauvages qui, rendus avec le temps dans la langue de l'homme policé, signifient et signifieront éternellement: justice, injustice ${ }^{22}$.

Ce qui distingue l'approche diderotienne d'autres approches, nous semble-t-il, c'est, à ce stade, le fait qu'elle insiste sur la dimension cognitive de

21. Colas Duflo, Diderot philosophe, Paris, Champion, 2003, p. 239.

22. Discours sur la poésie dramatique, ch. 10, Versini, IV, p. 1300 [Nous soulignons]. 
l'image ainsi produite. On voit par là ce qui amène Diderot - indépendamment des contingences historiques - à accorder une place prépondérante à l'art dans sa propre philosophie. Tout art chez Diderot consiste à rendre disponible à autrui le point de vue que l'on incarne, de sorte que la liaison des idées, qui sert ici de principe d'organisation, ne désigne que leur liaison dans l'entendement. Diderot ne suggère pas une "méthode d'exposition » qui reproduise la marche nécessaire de la nature, mais celle, contingente, d'un esprit: c'est qu'il s'agit de suivre l'ordre de l'invention, lequel n'est pas celui de la nature, mais celui, subjectif, qui représente la nature perçue. S'il s'agit de faire voir ce qu'on a découvert, comme c'est le cas dans toute théorie de la découverte, ce n'est pas ce qui était déjà là, mais recouvert, qu'on doit montrer, mais ce qui n'existe qu'autant qu'il a été perçu depuis la position qu'on occupe soi-même.

De cette façon, Diderot substitue à toute méthode le travail concret du dialogue: chaque organisation, par sa particularité, exprime la totalité du réel d'une manière différenciée; toutes les pratiques recèlent une possibilité d'accroître notre puissance perceptive par l'exercice qu'elles font faire. De là vient qu'il n'y a que la cumulation des points de vue - et ici prend sens un projet comme celui de l'Encyclopédie - qui puisse se constituer en véritable projet de connaissance. Sylvain Auroux le dit admirablement:

L'homme qui est le centre d'où le monde est connu, et à partir duquel il est raisonnable de totaliser la représentation incomplète de l'univers, ce n'est ni un individu, ni une rationalité abstraite, suspendue pour l'éternité, à un point fixe hors du monde, c'est l'humanité concrète, qui travaille, jouit et meurt ${ }^{23}$.

\section{Productions de l'esprit, fables et suites de pensées}

Le second aspect fondamental des productions de l'esprit dans la pensée moderne tient à un autre fait. Bien qu'elles s'efforcent de découvrir quelque chose, elles sont le plus souvent des objets qui ne représentent rien, des choses qui n'ont pas de modèles dans la nature, des inventions qui masquent ce qu'elles cherchent à rendre: des romans, des fables. Fontenelle pourra donc écrire, en ouverture de L'Origine des Fables:

23. Sylvain Auroux, «Diderot encyclopédiste », Stanford French Review, automne 1984, p. 185, qui ajoute: «Le coup de génie du philosophe consiste donc à avoir accepté de laisser le savoir à lui-même, à sa positivité et à sa pratique. » (p. 186.) 
Les Fables des Grecs n'étaient pas comme nos Romans qu'on nous donne pour ce qu'ils sont, et non pas pour des Histoires; il n'y a point d'autres Histoires anciennes que les Fables. Éclaircissons, s'il se peut, cette matière; étudions l'esprit humain dans l'une de ses plus étranges productions: c'est là bien souvent qu'il se donne le mieux à connaître ${ }^{24}$.

Le nom de roman ou de fable désigne ici soit une production qui ne représente rien de réel et qui est donnée comme telle - un objet nouveau, et, pour cette raison, généralement considéré comme futile, sans autre intérêt que de pur divertissement -, soit une production qui ne représente rien de réel, mais qui y prétend - objet nouveau, encore, mais cette fois considéré comme dangereux, parce qu'instituant un faux savoir.

On aurait tort, cependant, de réduire la fable à une production fausse prise dans une simplissime dichotomie discours vrai/discours faux. Le traité de Fontenelle sur L'Origine des Fables est au contraire entièrement tourné vers la démonstration de leur nécessité historique: les fables sont les «systèmes " philosophiques d'époques révolues. Elles ne pouvaient pas se donner pour romans, parce qu'elles étaient prises pour des représentations de la réalité. Tout l'enjeu de la pensée historicisante de Fontenelle consiste précisément dans cette prise de position typiquement «moderne " (au sens que donne à ce terme la Querelle des Anciens et des Modernes), modulée par un refus de considérer le progrès accompli comme un saut en dehors de la fable. La philosophie, bien qu'elle se raffine, bien qu'elle parvienne à des résultats de plus en plus probants, garde toujours quelque chose en son centre qui la rapproche d'un processus de métaphorisation.

Cette Philosophie des premiers siècles, écrit-il, roulait sur un principe si naturel, qu'encore aujourd'hui notre Philosophie n'en a point d'autre [...] Nous expliquons les choses inconnues de la nature par celles que nous avons devant les yeux, et $[\ldots]$ nous transportons à la physique les idées que l'expérience nous fournit ${ }^{25}$.

L'un des aspects cruciaux de cette explication de l'origine des fables est cette invariance du principe sur lequel « roule » la philosophie. Celle-ci s'organise toujours sur la base d'un raisonnement analogique qui fait de toute connaissance une sorte de modélisation des phénomènes observés, laquelle permet de constituer des explications par transfert à d'autres phénomènes. C'est là un caractère fondamental de la théorie de la connaissance chez

24. De l'origine des Fables dans Euvres Complètes (A. Niderst éd.), Paris, Fayard (Corpus des œuvres de philosophie en langue française), 1991, vol. III, p. 187.

25. Ibid., p. 189. 
Fontenelle que de reposer sur cette puissance de composition de l'esprit. Ici, le savoir, s'il est toujours pensé sur l'horizon d'une imitation, n'a plus le caractère de pure intuition qu'il a chez Descartes. Il relève plutôt d'une projection: l'esprit produit bel et bien un système sur la base de phénomènes dont il « devine » les causes ${ }^{26}$. Découvrir est le résultat d'une anticipation, c'est-àdire d'une activité résolument créatrice de l'esprit. L'imagination est ainsi mise à profit comme une ressource cognitive primordiale, puisque c'est en elle que la philosophie d'une époque se constitue comme une configuration concrète exprimant l'état du savoir - et qu'elle se constitue comme une fable.

En ce sens, la fable est une hypothèse; le savoir issu du raisonnement est ouverture sur le possible - ce qui d'entrée de jeu donne toute sa force à cet élan vers la "pluralité des mondes » chez Fontenelle. C'est là un thème qui, sous divers auspices, est disséminé dans les diverses figures de l'épicurisme moderne ${ }^{27}$ - de Cyrano de Bergerac à Fontenelle, voire jusque dans le matérialisme hobbesien.

Rappelons en effet que Hobbes fait de l'invention une fonction de l'imagination et de la «suite dirigée des pensées », c'est-à-dire du « discours mental »:

Le discours mental, quand il est ordonné à un dessein, n'est rien d'autre que la recherche (ou faculté d'inventer que les latins appellent sagacitas et solertia), l'investigation des causes d'un effet quelconque présent ou passé, ou bien des effets d'une cause quelconque présente ou passée ${ }^{28}$.

Les figures de ces suites de pensée sont le souvenir et la prudence ou prévision. La prudence définit une capacité d'anticiper sur l'avenir, de produire une représentation de ce qui n'est pas encore, à partir du connu. Le passage de cette forme de savoir à la forme supérieure qu'est la science n'est en fait qu'une extension de ce pouvoir d'anticipation, extension qui est rendue possible par la production des signes. C'est dans la matière du langage que les suites de pensées particulières deviennent des théorèmes ${ }^{29}$. Mais le pouvoir

26. «Le philosophe a devant lui un certain nombre d'effets de la nature et d'expériences; il faut qu'il en devine des causes vraisemblables, et que de ce qu'il voyoit, et de ce qu'il devine, il en compose un tout bien lié; voilà le systême. L'Historien a aussi un certain nombre de faits dont il imagine les motifs, et sur lesquels il bâtit le mieux qu'il peut un systême d'histoire, plus incertain encore et plus sujet à caution qu'un systême de philosophie. " (Sur l'histoire, Euvres Complètes, op. cit., III, p. 176)

27. Sur la place de l'hypothèse dans l'épicurisme antique, voir Marcel Conche, Lucrèce, Paris, Seghers, 1967, p. 30-35.

28. Thomas Hoвbes, Léviathan (traduction, introduction, notes et notices de G. Mairet), Paris, Gallimard (Folio essais), 2000, p. 88.

29. Ibid., p. 115. 
de l'imagination, chez Hobbes, celui qui assure la sortie de l'expérience immédiate, est aussi responsable de l'erreur:

Calculer sans utiliser de mots, ce qui peut se faire pour des choses particulières (ainsi quand, à la vue d'une chose quelconque, nous conjecturons ce qui lui a probablement précédé, ou ce qui va probablement lui succéder), si ce qu'on pensait probablement succéder ne succède pas, ou ce qu'on pensait probablement avoir précédé n'a pas précédé, alors cela s'appelle ERREUR ${ }^{30}$.

L'erreur provient d'une déviation du pouvoir d'invention. En effet, en cherchant à composer à partir d'expériences passées une chaîne d'images dont la résolution doit être la représentation d'une cause qui n'a pas fait l'objet d'une expérience ou d'une conséquence attendue, l'invention parvient à la production d'une fiction qui n'est rien d'autre qu'une image caractérisée par son écart par rapport à la réalité.

Dès lors, la faculté de raisonner constitue toujours une activité productrice; c'est pourquoi Hobbes conclura que les suites de pensées, quand elles s'aventurent au-delà de l'expérience sensible, ne peuvent être considérées comme des connaissances absolues.

Aucun discours, quel qu'il soit, ne peut se terminer dans la connaissance absolue d'un fait passé ou à venir. En effet, pour ce qui est de la connaissance d'un fait, elle a son origine dans la sensation et, à la suite de cela, dans la mémoire. Et, en ce qui concerne la connaissance des conséquences, dont j'ai déjà dit que c'est ce qu'on appelle science, elle n'est pas absolue, mais conditionnelle ${ }^{31}$.

Le caractère productif de l'esprit, parce qu'il s'explique non comme déploiement analytique de ce qu'il contient déjà, mais comme puissance d'anticiper, est conçu comme une avancée aveugle dans le champ du possible. Le résultat de ses opérations est toujours, en dernière instance, affecté d'une dimension hypothétique: les enchaînements d'images dans l'imagination produisent par composition une représentation particulière dont la nature de découverte est, pour ainsi dire, mise en attente de confirmation par une chaîne correspondante d'événements; les enchaînement de mots constituent des chaînes déductives dont les conclusions sont conditionnelles, c'est-à-dire s'expriment dans la forme «si... alors... ".

Ces deux tendances interprétatives de l'épicurisme, dont l'une met l'accent sur la structure logique du raisonnement et son rapport à l'expérience,

30. Ibid., p. 114 [Nous soulignons].

31. Ibid., p. 142 [Nous soulignons]. 
l'autre sur la constitution historique de systèmes sont à prendre en considération pour comprendre la théorie de la science chez Diderot. Dans un premier temps, Diderot, a fortement insisté sur le caractère conjectural de tout raisonnement. Toute sortie hors de la pure expérience sensible a, pour Diderot, une forme "prudentielle». Cela découle, en partie, de sa conception de l'invention: dans la mesure où toute idée est une interprétation d'un phénomène, elle n'a aucun autre support que l'esprit qui perçoit, elle demeure essentiellement une instance subjective. L'article IGNORAncE le dit clairement:

Le défaut de nos sens ne nous laisse que des conjectures, fondées sur des idées qui sont peut-être fausses, \& nous ne pouvons être assurés d'aucune chose sur leur sujet, que de ce que nous pouvons en apprendre par un petit nombre d'expériences qui ne réussissent pas toujours, \& dont chacun explique les opérations secrettes à sa fantaisie ${ }^{32}$.

La nature ne s'offrant que de manière fragmentaire, son unification dans la perception, puis l'ordonnancement et la liaison de ces perceptions, qui n'ont d'autre principe que la spontanéité de notre organisation et la facticité de l'ordre, doivent être considérés comme des arrangements possibles des choses. La raison, chez Diderot, n'est pas moins tâtonnante que la pratique. Dit autrement: sa capacité d'outrepasser le donné sensible en fait une source d'erreur par excellence.

Une des principales différences de l'observateur de la nature et de son interprète, c'est que celui-ci part du point où les sens et les instruments abandonnent l'autre; il conjecture, par ce qui est, de ce qui doit être; il tire de l'ordre des choses des conclusions abstraites et générales, qui ont pour lui toute l'évidence des vérités sensibles et particulières ${ }^{33}$.

La théorie de la conjecture, chez Diderot, travaille sur deux plans. Sur le plan normatif, elle définit la fonction du raisonnement scientifique à la source des théories générales et des résultats attendus d'une expérience. Elle est alors une structure d'anticipation. Elle produit par avance ce qui reste à découvrir. Sur le plan descriptif - par où Diderot rejoint les Fontenelle et les Bayle qui lui ont ouvert la voie - elle explicite la façon dont s'est déroulée l’histoire de la philosophie.

Les phases différentes de la Lune sont, à ce qu'on dit, les premiers phénomenes célestes dont ils furent frappés; $\&$ en effet les inconstances de cet astre me

32. Article Ignorance [Diderot], Encyclopédie, VIII, p. 649.

33. Pensées sur l’interprétation de la nature, §56, Versini, I, p. 593. 
semblent plus propres à incliner les hommes à la méditation, que le spectacle constant du Soleil, toûjours le même sous un ciel toûjours sérain. Quoique nous ayons l'expérience journaliere de la vicissitude des êtres qui nous environnent, il semble que nous nous attendions à les trouver constamment tels que nous les avons vûs une premiere fois; \& quand le contraire est arrivé, nous le remarquons avec un mouvement de surprise: or l'observation \& l'étonnement sont les premiers pas de l'esprit vers la recherche des causes. Les Ethiopiens rencontrerent celle des phases de la Lune; ils assûrerent que cet astre ne brille que d'une lumiere empruntée. Les révolutions \& même les irrégularités des autres corps célestes, ne leur échapperent pas; ils formerent des conjectures sur la nature de ces êtres; $i l s$ en firent des causes physiques générales. Ils leur attribuerent différens effets, \& ce fut ainsi que l'Astrologie naquit parmi eux de la connoissance astronomique ${ }^{34}$.

L'intérêt de Diderot, sur ce point, consiste à sortir du simple régime d'opposition superstition/savoir, en montrant leur homologie de structure: dès lors que le savoir a été défini comme l'effet d'une véritable production, on ne peut plus le prendre dans ce genre de réduction. Les constructions successives qui font de la philosophie une aventure historique n'offrent pas de prise à un regard surplombant qui donnerait la garantie d'un "progrès de l'esprit humain ». Si progrès il y a, son statut de production sans autre ancrage dans le réel que l'esprit de ceux qui le porte est chose fragile: l'Encyclopédie se présente comme un recours technique pour combler ce déficit de stabilité dans l'immanence d'une pratique s'effectuant dans et pour l'histoire humaine. Elle fonde un lieu d'échange où l'objectivation du savoir ne se réalise que peu à peu, et ce lieu d'échange court éternellement le risque de retomber dans la barbarie, quels que soient les acquis.

\section{Productions de l'esprit, abstraction et modèle idéal}

Dans un tel contexte, la formation des concepts ne peut plus s'entendre comme une perception ou une intuition. Toute idée ou notion procède d'un processus inaperçu d'abstraction. Le principe des indiscernables montre en effet pour Diderot que ce qui nous fait prendre des choses pour des objets susceptibles d'être identifiés vient de ce que nos perceptions ne sont pas assez distinctes. Nous les rangeons dans des classes, parce que nous ne voyons pas que la nature est toute de nuances.

Ainsi, l'abstraction provient-elle entièrement de la construction d'une forme prélevée sur un contenu d'expérience. Le couplage hobbesien d'em-

34. Article Éthiopiens, Encyclopédie, VI, p. 55 [Nous soulignons]. 
pirisme épicurien et de nominalisme ouvre une brèche dans la conception scolastique de la connaissance: la connaissance moderne n'est pas contemplation, découverte de la réalité, mais constitution d'un système de signes. Les termes abstraits, on s'en souviendra, désignent les seules propriétés communes à plusieurs individus:

Un nom universel est attribué à plusieurs choses à cause de leur qualité semblable ou de quelque autre accident. Et, alors qu'un nom propre amène à l'esprit une action seulement, les universaux rappellent à l'esprit n'importe laquelle de ces choses multiples ${ }^{35}$.

Ayant posé en principe que «nous n’imaginons que ce dont nous avons eu précédemment la sensation » ${ }^{36}$, Hobbes peut ainsi montrer qu'à notre incapacité d'avoir des idées abstraites - au sens où pourrions en avoir une intuition -, nous substituons un mot, dont le support est, dans l'imagination, un individu appartenant à la classe désignée par le terme. C'est en ce sens que la connaissance n'est ici qu'une constitution d'un système de signes: l'esprit produit un signe qui lui rappelle une opération de liaison qu'il a faite entre deux individus ou l'opération abstractive par laquelle il a isolé une propriété d'un individu. Ce type de nominalisme, jusqu'à un certain point, ouvre la voie aux analyses de Condillac sur une langue des calculs, c'est-à-dire de signes à partir desquels l'analyse intégrale des phénomènes devient possible.

Les invectives hobbesiennes contre ceux qui professent l'existence d' "essences séparées " ${ }^{37}$ dérivent directement de ce nominalisme. Le réalisme des essences est fondé sur une mécompréhension des procédures d'abstraction.

C'est dans cette veine aussi que s'inscrit l'entreprise de Locke. Pour lui, il s'agit de montrer, par une explication empiriste, la genèse de nos idées, et elle aboutit à conclure que les idées abstraites sont réductibles à des actes cognitifs:

Lors donc que nous laissons à part les particuliers, les généraux qui restent, ne sont que de simples productions de notre esprit, dont la nature générale n'est autre chose que la capacité que l'entendement leur communique, de signifier ou de représenter plusieurs particuliers ${ }^{38}$.

35. Thomas Hoвbes, Leviathan, op. cit., p. 99.

36. Ibid., p. 84.

37. Cf. Hobbes dans le chapitre 46 du Léviathan, intitulé « Des ténèbres produites par la vaine philosophie et les traditions fabuleuses ", op. cit., p. 907-937.

38. John Locke, Essai philosophique concernant l'entendement humain (traduction de P. Coste, édition de G. J. D. Moyal), Paris, Champion, 2004, Livre III, ch. 3, § 11. 
Cependant, dans le schéma généalogique de Locke, la production des idées abstraites s'explique d'une manière qui permet de faire l'économie de toute fonction cruciale de l'imagination: la réflexion est, pour ainsi dire, donnée d'emblée ${ }^{39}$, l'entendement peut tirer immédiatement des particuliers des propriétés isolées. Ce qui intéresse Locke chez Hobbes, c'est la faculté qu'a l'esprit humain de produire un signe qui lui rappelle une opération qu'il a faite antérieurement.

Cette forme de nominalisme fait cependant difficulté: la production de l'esprit demeure entièrement pensée à travers le prisme de ce que l'on pourrait appeler un dualisme sémantique. L'abstraction, dans la mesure où l'on refuse qu'elle découle d'une perception de forme substantielle présente dans l'individu, est un acte de prélèvement analytique, par l'entendement, d'une propriété d'un particulier. Le terme abstrait ainsi forgé désigne alors quelles propriétés sont considérées comme significatives dans ce particulier pour le faire entrer dans la classe désignée par ce terme: telle figure rouge sera appelée "triangle » si elle a trois côtés, et sera ainsi désignée par cette propriété que l'on juge significative. Le terme abstrait est dès lors considéré comme une production de l'esprit au sens où il s'agit d'une réification dans un terme associé à l'acte même par lequel la propriété qu'il désigne a été isolée. Toutefois - et c'est bien là ce que dit Hobbes -, cela signifie que les termes abstraits ne sont pas des productions d'objets nouveaux, mais des répétitions du matériau sensible dans la forme de la pensée. Même si la pensée elle-même n'est pas considérée comme le lieu de l'intuition de quelque fondement suprasensible du monde matériel, l'invention qui résulte de son activité est entièrement déterminée par une décomposition qui a pour fin l'abstraction. En d'autres termes, la structure sémantique du terme abstrait est pensée comme une relation objective entre la manifestation sensible d'une propriété et son expression abstraite, relation, donc, qui n'est pas proprement produite par l'esprit, mais perçue, découverte. Que le matériau de la pensée ne soit pas fait d'intuitions intellectuelles de la structure suprasensible du monde matériel n'enlève rien à l'idée que le concept - fût-il un mot - tient la place, dans l'esprit, de quelque chose de réel. C'est pourquoi la connaissance d'une chose individuelle est considérée plus distincte si chacune de ses propriétés a été isolée ${ }^{40}$.

39. Ibid., Livre I, ch. 1, $\S 2$.

40. Condillac écrit (Essai sur l'origine des connaissances humaines, $1^{\text {re }}$ partie, sect. $5, \S 12$, dans Euvres philosophiques (G. Leroy éd.), Paris, PUF (Corpus général des philosophes français), 1947-1951, I, p. 52): « Les noms des substances tiennent dans notre esprit la place que les 
Dans son Abrégé de la philosophie de Gassendi, François Bernier commence par un chapitre "De la simple imagination des choses en XVIII règles " ${ }^{41}$. L'imagination y est définie de façon qui n'est pas strictement mnémonique:

Nous prenons icy le mot d'Imagination pour la Pensée, ou l'action de l'Entendement qui se termine à l'image de la chose pensée, à l'image, dis-je, que l'Entendement semble regarder, et avoir, pour ainsi dire, devant ses yeux lors qu'il pense à quelque chose ${ }^{42}$.

Or cette Image qui lorsque nous pensons à quelque chose est presente à l'Entendement, et est comme son object, a aussi plusieurs autres noms: Car elle est appelée Idée, et Espece, et en luy accommodant le nom d'action, elle est dite Notion, Prenotion, Anticipation, ou notion anticipée, et deplus Concept, et Phantome, entant qu'elle a son siege dans la Phantaisie ou faculté Imaginatrice ${ }^{43}$.

Dans les 18 règles qui suivent ce préambule, Bernier veut montrer que l'imagination est non pas le lieu où n'importe quel objet singulier se présente par un mouvement de l'entendement vers l'idée abstraite de la classe à laquelle cet objet appartient, mais bien la pensée elle-même, c'est-à-dire le lieu où toute action de l'esprit se termine - lieu, dirions-nous, où les matériaux de l'esprit sont, au terme d'un acte cognitif, rassemblés en cette image unifiée que forme l'idée, le concept. Ainsi, la première règle dit: " la simple imagination d'une chose est telle que l'idée qu'on a de la chose ${ }^{44}$. " Les autres règles montrent que, si nos premières idées viennent des sens, et si, par différentes opérations, l'entendement parvient peu à peu à en former de nouvelles qui, toutes, finissent en une image dans l'imagination; celle-ci est non pas quelconque, mais coordonnée en vue de représenter l'idée qu'on a de la chose. L'activité de l'entendement se termine toujours dans la production d'un objet sensible unifié. Alors, soit la recherche des causes d'un phénomène conduit à une anticipation ${ }^{45}$, soit, dans le cas de l'abstraction, elle

sujets occupent hors de nous: ils y sont le lien et le soutien des idées simples, comme les sujets le sont au dehors des qualités. " Rien ne montre mieux cette persistance de la pensée de Condillac à penser l'unité du concept comme formellement représentative de l'unité des objets hors de nous, c'est-à-dire non comme une production d'un esprit qui a besoin d'ordonner ses perceptions pour connaître, mais comme celle d'un esprit qui, par sa passivité première, reproduit selon son mode de réception propre les objets qu'il perçoit.

41. François Bernier, Abrégé de la philosophie de Gassendi (texte établi par S. Murr),

Paris, Fayard (Corpus des oeuvres de philosophie en langue française), 1992, p. 25.

42. ID.

43. Ibid., p. 26.

44. Ibid., p. 27.

45. Jean-Charles Darmon, «Sortir du scepticisme: Gassendi et les signes », Pierre-François Moreau (dir.), Le Scepticisme au XVI et au XVII esiècle, Paris, Albin Michel, 2001, p. 222-238. 
épure la représentation pour produire une idée qui convient aux individus et aux qualités désignés par le terme abstrait. On change, alors, de registre : la logique devient une sorte de psychologie descriptive; les idées que nous formons dépendent des connaissances empiriques que nous avons acquises ${ }^{46}$.

Il y a là, si l'on veut, un travail continu de synthèse opérée par l'imagination qui assure que la progression vers les idées plus générales coordonne un procès d'idéalisation dans l'imagination-même qui fournit ainsi, à chaque étape, une "image " résultant des opérations de l'entendement. L'imagination a donc un rôle qui n'est pas simplement de mémorisation dans le développement des connaissances ${ }^{47}$. L'imagination construit des ressemblances et, atteignant les limites de ce qu'elle peut présenter, figure l'infigurable par le jeu d'accommodement du transport métaphorique:

Et c'est mesme aussi de cette maniere que l'Entendement, tant qu'il est uni au corps, a coutume de concevoir Dieu, qui ne peut assurement point tomber dans le Sens, sous l'idée de quelque Vieillard venerable qu'on aura veu, ou de quelque grand Roy environné de gloire, et de majesté, ou de quelque Lumiere tres eclatante, luy accommodant en quelque façon quelqu'une de ces idées ${ }^{48}$.

Dans la mesure où toute activité de l'esprit doit se résoudre en une image singulière, l'imagination est comme poussée hors de sa pure activité mnémonique qui l'empêcherait de figurer autre chose que ce dont elle a eu la sensation, sa puissance synthétique l'amène à concevoir ce qui ne tombe pas sous le sens, dans une figure qui exprime le rapport sous lequel nous le concevons. Certes, cette figure reste imparfaite: mais son imperfection n'est pas liée à l'écart qui existerait entre l'idée dans l'esprit (intuition intellectuelle, terme abstrait) et sa figuration dans l'imagination, puisque idée et figures sont réductibles l'une à l'autre; son imperfection serait simplement signe de l'écart irréductible entre l'idée et l'objet qu'elle dénote. Dans une

46. "Car comme elle est dite generale, premierement par assemblage, en ce que c'est un Amas qui contient toutes celles qui sont de mesme Genre; elle sera sans doute d'autant plus parfaite, et plus complete qu'il luy en manquera moins. Desorte que si quelqu'un dans l'idée qu'il a des Hommes, comprend non seulement les Européens, les Africains, et les Asiatiques, mais aussi les Américains, il aura cette idée plus parfaite, que si à la façon des Anciens il n'y comprenoit que les seuls Européens, les Africains, et les Asiatiques. » (François BERNIER, Abrégé de la philosophie de Gassendi, op. cit., p. 38.)

47. Annie BECQ (op. cit., p. 127) : «La pensée travaille sur les synthèses effectuées par l'imagination, en un processus continu d'interprétation de ces » données «sensibles, ordonné à l'expérience humaine..."

48. François BERNIER, op. cit, p. 29. 
telle sémantique, il n'y a plus d'écart entre l'Idée ou Image qui se présente à l'imagination et le sens du terme que nous lui attachons; il n'y a plus d'écart dans le sujet entre ce qu'il pense et ce qu'il imagine. Le concept, alors, n'est pas une imitation de la chose, mais son expression, une manière, pour cette chose, de se présenter.

Dans la théorie diderotienne de l'imagination, la notion de tableau est importante: Diderot jugeait en effet que la définition de la justice devait à terme pouvoir se décomposer en une image où les rapports que désigne le terme «justice» seraient sensibles. Cette idéen'est pas spécifique à Diderot: elle est courante dans le sensualisme français et relie les apports de la théorie empiriste de la connaissance à la tradition rhétorique ${ }^{49}$. Toutefois, cette notion joue chez Diderot une fonction essentielle, puisqu'elle constitue le point nodal de toute sa théorie des concepts et donc de la productivité de l'esprit. Le tableau représente la façon dont le multiple se présente sous la forme de l'unité: si une âme avait mille bouches disant simultanément ce qu'elle ressent, ce serait un tableau verbal du tableau mouvant qu'elle est elle-même. L'unité, ici, désigne évidemment le fait que cette multiplicité est réunie sous la forme d'un concept ou d'un sens; or, comme le sens est le résultat de cette multiplicité, le tableau représente très exactement l'action de cette multiplicité et sa façon de s'agencer en une unité. En d'autres termes, la perception sensible s'agence en un tableau dans l'âme, et la façon dont l'âme se rapporte aux divers éléments de cette perception décrit le degré de connaissance analytique qu'elle a de ce tableau, à savoir la vivacité avec laquelle chacun de ces éléments apparaît. Si cela vaut pour le tableau qui se peint dans l'âme, on doit admettre que l'imitation de ce tableau en constitue la meilleure expression possible. C'est pourquoi, en un sens, tant l'épistémologie que l'esthétique de Diderot sont orientées vers ce qui apparaît comme une théorie de l'imitation. Mais, comme le tableau représente toujours un acte cognitif subjectif producteur d'un concept, l'imitation consiste en une objectivation des liaisons opérées subjectivement au moment de la formation dudit concept.

49. Pierre Frantz, L'esthétique du tableau dans le théâtre du XviII siècle, p. 7-20. Voir aussi Condillac, De l'art d'écrire, Livre II, ch. 1, Euvres philosophiques, op. cit. I p. 551 : «Il faut considérer une pensée composée comme un tableau bien fait, où tout est d'accord. Soit que le peintre sépare ou groupe les figures, qu'il les éloigne ou les rapproche; il les lie toutes par la part qu'elles prennent à une action principale. Il donne à chacune un caractère; mais ce caractère n'est développé que par les accessoires qui conviennent aux circonstances. Il n'est jamais occupé d'une seule figure; il l'est continuellement du tableau entier; il fait un ensemble où tout est dans une exacte proportion." 
L'analyse des mécanismes à l'œuvre dans la production des concepts fait voir que le concept d'un phénomène n'est rien d'autre que l'expression du résultat de l'acte intentionnel par lequel ce phénomène est appréhendé par l'esprit. Dès lors, le tableau lui-même doit être compris comme une telle expression, c'est-à-dire, non du phénomène, mais de l'acte cognitif par lequel ce phénomène est constitué en concept. Le tableau est un concept, une vue de l'esprit, présentant les marques sur lesquelles l'acte perceptif s'est appuyé pour forger le sens du phénomène. Le tableau, en somme, est l'expression du travail de la subjectivité sur le phénomène. Ainsi, dans le tableau de l'injustice, Diderot dit bien que ce qui importe, c'est qu'y soient lisibles en même temps toutes les instances relationnelles qui, à être perçues, produisent l'affect que nous avons antérieurement lié à la justice. Les deux hommes du tableau, peu importent leurs caractéristiques particulières (conformation physique, vêtements, rang social, accessoires, etc.), doivent minimalement arborer certaines expressions de la douleur et de la culpabilité pour permettre la saisie du rapport (entre ces « impressions contraires", ces "mouvements opposés») dont le concept de justice est l'expression. L'artiste - mais Diderot assure que c'est là aussi la tâche du philosophe doit donc focaliser son attention (et attirer celle de ses interlocuteurs) sur les idées qui ont été liées et dont l'intrication est exprimée par le concept. Il doit, en somme, réinventer le concept, redécouvrir ce qui a été découvert lors de sa formation, penser par lui-même ce en direction de quoi le concept donne à penser, s'il part d'un "sujet » donné; ou produire un concept nouveau, donner à voir les rapports qu'il a aperçus ${ }^{50}$. On voit par là à quel point Diderot participe au déplacement qui affecte la notion d'imitation de la nature au XVIII ${ }^{\mathrm{e}}$ siècle ${ }^{51}$, il cherche à en dégager un sens profondément gnoséologique. L'importance qu'il confère à la nécessité d'un redéploiement des conditions sensibles au sein desquelles s'effectue la perception de rapports permet de mesurer la place grandissante qu'il donnera à l'imagination comme faculté par excellence sur laquelle doit s'appuyer cet effort d'invention dont découle notre « interprétation de la nature », c'est-à-dire la constellation de concepts que nous produisons pour cerner la prolifération sans cesse différenciée de la nature.

50. «Quand on a de la verve, des concepts rares, une manière d'apercevoir et de sentir originale et forte, le grand tourment est de trouver l'expression singulière, individuelle, unique, qui caractérise, qui distingue, qui attache et qui frappe. " (Salon de 1767, Versini, IV, p. 666 [Nous soulignons]).

51. Annie BECQ, "Splendeur et misère de l'imitation au siècle des Lumières 》, dans Ulla Kölving et Irène Passeron (dir.), Sciences, musique, Lumières: mélanges offerts à AnneMarie Chouillet, p. 385-391. 
Cette théorie du tableau trouvera l'un de ses termes les plus significatifs dans la théorie du modèle idéal du Salon de 1767. Un jeu ironique sur les catégories de Platon sert alors à Diderot à montrer comment l'imitation est productive de connaissance ${ }^{52}$. Voici le point essentiel de la démonstration ${ }^{53}$ : pour Diderot, l'artiste ne peut prétendre imiter les choses telles qu'elles sont: elles contiennent tellement de traits particuliers, qu'aucune peinture ne peut le faire. L'artiste, même sur modèle, ne copie pas, parce que son modèle n'est lui-même que le «fantôme » de la « ligne vraie » qui, elle, n'est que dans $s a$ tête. La ligne vraie désigne la vérité non en ce qui concerne ce qui se trouve devant l'artiste, mais en ce qui concerne l'idée qu'il en tire, c'est-à-dire l'effet de cette chose sur lui. La ligne vraie désigne donc l'ensemble des éléments de l'objet matériel qui sont mis en rapport pour qu'une chose soit conceptualisée de telle ou telle façon. L'un, devant un individu, voit un "homme de lettres», et en fait le portrait à partir des propriétés communes à cet individu et au "modèle idéal "sculptural de l'homme de lettres; un autre voit dans le même individu son "ami " et sculpte selon la même règle. La ligne vraie est - et c'est là que se révèle le revirement ironique des catégories platoniciennes - le résultat d'une invention de l'artiste. L'artiste peint non la chose telle qu'elle est, mais la «chose générale »:

Vous avez senti la différence de la chose générale et de la chose individuelle jusques dans les moindres parties, puisque vous n'oseriez pas m'assurer depuis le moment où vous prîtes le pinceau jusqu'à ce jour, de vous être assujetti à l'imitation rigoureuse d'un cheveu ${ }^{54}$.

«Voilà l'être imaginaire que vous devez prendre pour modèle. Plus fortement vous l'aurez conçu, plus vous serez grand, rare, merveilleux, sublime. " 55 Que la "ligne vraie " soit un être imaginaire montre que l'artiste, au même titre que le scientifique, procède de manière abstractive: il «ajoute " ou "supprime », et tire ainsi des objets matériels ses concepts. Sur le plan matériel, cette présentation de l'œuvre d'art répond à l'idée selon laquelle celle-ci est une imitation d'un acte à la fois cognitif et productif.

52. Il ne s'agit pas ici simplement de dire que Diderot a une esthétique cognitiviste: depuis Aristote, une telle esthétique existe. Nous voulons plutôt mettre l'accent sur cette dimension productive, qui suppose que l'œuvre ne forme pas qu'une représentation, c'est-à-dire le passage d'une substance idéelle dans une substance matérielle, ou le véhicule d'un agent transcendant (l'idée, le représenté, etc.), mais est en elle-même une «force », c'est-à-dire quelque chose qui doit être considéré comme un objet qui modifie les êtres qui le perçoivent.

53. Pour ce qui suit, voir Salon de 1767, Versini, IV, p. 522-524.

54. Ibid., Versini, IV, p. 522. 


\section{Conclusion}

Ce trop bref tour d'horizon permet déjà d'illustrer certains des aspects originaux de Diderot. D'abord, il semble clair que le sensualisme qu'il professe, étant donné le postulat matérialiste qui le fonde, l'arrache au pur nominalisme qui s'accommode généralement d'un certain réalisme. Diderot est conduit à mettre l'accent sur le fait que les particuliers sont eux-mêmes de purs concepts - ce qui le fait pencher vers un phénoménalisme plus radical que celui de la plupart de ses contemporains. Cela tend à donner une fonction primordiale à l'activité du sujet, mais d'un sujet qui n'est qu'une organisation différenciée, et au sujet duquel rien ne peut être établi de manière transcendantale. Cette absence de détermination transcendantale pourrait passer pour pur relativisme, ou scepticisme. Mais Diderot, nous semble-t-il, échappe à ce soupçon par sa manière de fonder dans la praxis un lieu d'élaboration, de stabilisation et d'échange des points de vue; il y a donc une objectivation de la connaissance: le savoir, en ce sens, est réellement conçu comme une activité de production. Cette façon de lire Diderot permet, à notre sens, de comprendre certaines des réticences qu'il a pu éprouver devant d'autres matérialismes - celui d'Helvétius, par exemple -, lesquels sont souvent, sur le plan épistémiques, incapables de justifier le réalisme sur lequel ils s'appuient et, maintiennent le savoir dans ce que nous avons appelé un dualisme résiduel. La pensée de Diderot pourrait bien être l'un des lieux les plus féconds du matérialisme d'Ancien régime pour penser rigoureusement la question de la productivité de l'esprit sur un plan d'immanence.

Une dernière remarque peut se faire. Le domaine qui appartient à l'elocutio classique apparaît donc comme celui où la connaissance atteint son degré de perfection analytique le plus élevé. Diderot s'oppose sur ce point à nombre de ses contemporains, parce qu'il juge que les figures n'ont pas l'imprécision que leur attribue, par exemple, Voltaire ${ }^{56}$, pour qui la seule clarté digne de la philosophie est la clarté rationnelle. En insistant sur les procé-

55. Ibid., Versini, IV, p. 528.

56. Article «Figuré, littérature», Encyclopédie, VI, p. 683: «L'imagination ardente, la passion, le désir souvent trompé de plaire par des images surprenantes, produisent le style figuré. Nous ne l'admettons point dans les histoires, car trop de métaphores nuisent à la clarté; elles nuisent même à la vérité, en disant plus ou moins que la chose même. Les ouvrages didactiques réprouvent ce style. Il est bien moins à sa place dans un sermon que dans une oraison funèbre; parce que le sermon est une instruction dans laquelle on annonce la vérité, l'oraison funèbre une déclamation dans laquelle on exagère. La poésie d'enthousiasme, comme l'épopée, l'ode, est le genre qui reçoit le plus ce style. On le prodigue moins dans la tragédie, où le dialogue doit être aussi naturel qu'élevé; encore moins dans la comédie, dont le style doit être plus simple. » 
dures de mise en scène, de divulgation du point de vue à partir duquel telle perception est rendue possible, Diderot renverse le classicisme et l'idéalisme sur lesquels il s'appuie. Plutôt que de juger nécessaire de domestiquer le champ de l'expérience par une maîtrise consciente par la raison, il juge qu'est une connaissance l'aptitude à en concevoir les conditions de reproductibilité: ce qui signifie produire un objet où les rapports qui sont « dérobés » dans un objet apparaissent et sont rendus «sensibles » dans un autre. De la sorte, l'esprit agit comme la nature elle-même:

Il est évident que la nature n'a pu conserver tant de ressemblance dans les parties et affecter tant de variété dans les formes, sans avoir souvent rendu sensible dans un être organisé ce qu'elle a dérobé dans un autre ${ }^{57}$.

Résumé: Dans cet article, nous nous intéressons à la façon particulière dont Diderot envisage le caractère productif de l'esprit en mettant certains aspects de son épistémologie et de son esthétique avec trois lieux théoriques hérités de ses prédecesseurs. D'abord, ce problème touche la question de l'invention en tant que méthode de construction du discours peu à peu assimilée à une méthode de découverte par Bacon et Descartes. Dans un second temps, il semble que Diderot, qui fait grand cas de la conjecture, définisse avant tout la connaissance comme une anticipation, prolongeant ainsi ce retour de l'épicurisme dans la pensée moderne. Enfin, du point de vue esthétique, Diderot développe une conception du modèle idéal qui s'accorde avec une théorie nominaliste de l'abstraction comme production d'un instrument conceptuel. Ces trois facettes de la pensée de Diderot contribuent, à notre sens, à la formation d'une véritable théorie matérialiste de la connaissance.

Mots-clés: Diderot. Connaissance. Invention. Imagination. Production de l'esprit.

Abstract:

Key words :

57. Pensées sur l'interprétation de la nature, § 12, Versini, p. 565 [Nous soulignons]. 